\title{
Correction to: Utility of pulmonary ultrasound to identify interstitial lung disease in patients with rheumatoid arthritis
}

\author{
Natalia Mena-Vázquez ${ }^{1,2}$ (1) - Francisco Gabriel Jimenez-Núñez ${ }^{1,2}$. Francisco Javier Godoy-Navarrete ${ }^{1,2}$. \\ Sara Manrique-Arija ${ }^{1,2}$. María Carmen Aguilar-Hurtado ${ }^{3}$. Carmen María Romero-Barco ${ }^{4}$. \\ Inmaculada Ureña-Garnica ${ }^{1,2}$ • F. Espildora ${ }^{5}$ - María Isabel Padin-Martín ${ }^{3}$ • Antonio Fernández-Nebro ${ }^{1,2,6}$
}

Published online: 18 April 2021

(C) International League of Associations for Rheumatology (ILAR) 2021

\section{Correction to: Clinical Rheumatology https://doi.org/10.1007/s10067-021-05655-1}

In the original published version of this article under the Result and Conclusion sections of the "Abstract" contained errors and has been corrected as follows [Bold text used to highlight problem area]:

Results: Seventy-one patients were included, 35 (49.2\%) with ILD-RA and 36 (50.8\%) RA controls. Regarding US score, we found that the detection of 5.5 lines in a reduced score of 8 intercostal spaces had a sensitivity $=62.2 \%$, specificity $=91.3 \%$, PPV $=88.4 \%$, and NPV $=69.5 \%$. In multivariate analysis, the intercostal spaces which showed independent association with ILD were 3rd right anterior axillary space (OR [IC 95\%] 19.0 [1.3-27.5]), 8th right posterior

The online version of the original article can be found at https://doi.org/ 10.1007/s10067-021-05655-1

Natalia Mena-Vázquez

nataliamenavazquez@gmail.com

$\triangle$ Francisco Gabriel Jimenez-Núñez cortesfra@gmail.com

1 Instituto de Investigación Biomédica de Málaga (IBIMA), 29010 Málaga, Spain

2 UGC de Reumatología, Hospital Regional Universitario de Málaga, Plaza del Hospital Civil s/n., 29009 Málaga, Spain

3 UGC de Radiodiagnóstico, Hospital Regional Universitario de Málaga, 29009 Málaga, Spain

4 UGC de Reumatología, Hospital Clínico Universitario Virgen de la Victoria, 29010 Málaga, Spain

5 UGC de Neumología, Hospital Regional Universitario de Málaga, 29009 Málaga, Spain

6 Departamento de Medicina, Universidad de Málaga, Málaga, Spain axillary space (OR [IC 95\%] 0.04 [0.0-0.6]), 8th right subscapular space (OR [IC 95\%] 16.5 [1.8-45.5]), 9th right paravertebral space (OR [IC 95\%] 7.11 [1.0-37.1]), and 2nd left clavicular middle space (OR [IC 95\%] 21.9 [1.26-37.8]). Conclusions: Lung ultrasound could be a useful tool for ILD diagnosis associated with rheumatoid arthritis. A 8-space reduced score showed a similar total predictive capacity than 72-space score.

The original article has been corrected.

Publisher's note Springer Nature remains neutral with regard to jurisdictional claims in published maps and institutional affiliations. 